\title{
The Quality of Toddler Child Care and Cognitive Skills at 24 Months: Propensity Score Analysis Results from the ECLS-B
}

\author{
Erik Ruzek $^{\mathrm{a}}$, Margaret Burchinal ${ }^{\mathrm{b}}$, George Farkas ${ }^{\mathrm{c}}$, and Greg J. Duncan ${ }^{\mathrm{c}}$ \\ Margaret Burchinal: burchinal@unc.edu; George Farkas: gfarkas@uci.edu; Greg J. Duncan: gduncan@uci.edu \\ aCenter for Advanced Study of Teaching and Learning, University of Virginia; 350 Old Ivy Way, \\ Suite 202, Charlottesville, VA 22903, USA \\ bFPG Child Development Institute, University of North Carolina; Campus Box 8185, Chapel Hill, \\ NC 27599-8185, USA \\ 'School of Education, University of California, Irvine; 3200 Education, Irvine, CA 92697-5500, \\ USA
}

\begin{abstract}
Over half of the toddlers in the U.S. experience routine nonparental care, but much less is known about early care than about preschool care. This study analyzed 2-year-old child care and child outcome data from the nationally representative ECLS-B sample of children born in 2001. At twoyears of age, $51 \%$ of children experienced exclusive parental care, $18 \%$ relative care, $15 \%$ family child care, and $16 \%$ center care. More children in nonparental care were in medium quality care $(61 \%)$ than in high quality $(26 \%)$ or low quality $(13 \%)$ care. Low-income children were more likely than non-low income children to be cared for by their parents and, when in care, were more often in lower quality care. The impact of toddler care quality on cognitive skills was estimated using propensity score adjustments to account for potential selection confounds due to family and child characteristics. Children's cognitive scores were higher in high or medium quality care than in low quality care, but no evidence emerged suggesting that poverty moderated the quality effects. Nevertheless, this suggests that increasing the proportion of low-income children in high quality care could reduce the achievement gap because low-income children are very unlikely to experience high quality care.
\end{abstract}

\section{Keywords}

toddler care; child care quality; ECLS-B; cognitive skills; propensity scores

\begin{abstract}
The care of infants and toddlers has undergone significant changes over the last 40 years. In decades past, the vast majority of 12-24-month-old children were cared for at home by their mothers. By the mid 2000's, however, less than half of infants and toddlers in the U.S. stayed at home with their mothers, indicating that the majority of 1- to 2-year-old U.S. children receive part- or full-time care by people other than their parents (Laughlin, 2010). Ensuring that infants and toddlers benefit from positive early experiences is important to
\end{abstract}

(c) 2013 Elsevier Inc. All rights reserved.

Corresponding author contact: eruzek@ virginia.edu; 350 Old Ivy Way, Suite 202, Charlottesville, VA 22903; 001-434-243-3412.

Publisher's Disclaimer: This is a PDF file of an unedited manuscript that has been accepted for publication. As a service to our customers we are providing this early version of the manuscript. The manuscript will undergo copyediting, typesetting, and review of the resulting proof before it is published in its final citable form. Please note that during the production process errors may be discovered which could affect the content, and all legal disclaimers that apply to the journal pertain. 
parents, and is of growing concern for policy makers. Since most child care research focuses on the preschool period, little is known about the impact of nonparental child care experiences during the infant and toddler period, especially at the national level. The purpose of this study is to extend our understanding of the impact of child care experiences for infants and toddlers by analyzing a nationally-representative data source on children's early development, the Early Childhood Longitudinal Study-Birth cohort of children born in 2001 (Flanagan \& West, 2005). Propensity score methods are used to reduce bias in the estimation of the effect of child care quality on the cognitive development of 2-year-old children owing to parental selection of different types and quality of child care.

\section{High Quality Child Care}

The quality of care received by infants and toddlers is thought to be crucial to their development, and in order to provide maximal developmental benefit, child-adult interactions must be continually sustained and grow increasingly complex over time (Bronfenbrenner \& Morris, 2006). Increasing complexity arises when caregiver-child interactions are scaffolded to reflect the child's understanding of relationships and objects. Constructionist theorists extend the interaction-based child care quality model to argue that young children construct their own learning by interacting with and operating in their natural environments (Bodrova \& Leong, 2006; Piaget, 2007; Vygotsky, 1978). These theories have been influential in shaping our understanding of the quality of child care, and specifically the importance of both the quality of adult-child interactions and the child's opportunity to interact with varied and rich materials within the child care classroom. In prior child care research, developmentally-supportive environments were found to be rich in spoken language experiences; encourage children to safely explore their physical, social, and intellectual environment; and contain play environments that stimulate children's cognitive development (National Institute of Child Health and Human Development, Early Child Care Research Network [NICHD ECCRN], 2000a; Vandell, 2004). We define high quality child care as care that occurs in safe settings where children are provided with rich play environments and reciprocal interactions that encourage exploration and learning.

\section{Child Care Quality and Children's Cognitive Development}

Studies of preschool children consistently find a positive association between child care quality and children's cognitive skills (Burchinal \& Cryer, 2003; Burchinal, Kainz, \& Cai, 2011; Camilli, Vargas, Ryan, \& Barnett, 2010; Gormley, Gayer, Phillips, \& Dawson, 2005; Howes et al., 2008; Mashburn et al., 2008; NICHD ECCRN, 2000a, 2002a, 2005; NICHD ECCRN \& Duncan, 2003; Peisner-Feinberg \& Burchinal, 1997; Peisner-Feinberg et al., 2001; Pianta, Barnett, Burchinal, \& Thornburg, 2009; Reynolds, Temple, Robertson, \& Mann, 2002; Vandell, 2004; Votruba-Drzal, Coley, \& Chase-Lansdale, 2004). However, much less is known about child care quality effects on infant and toddler cognitive development. Experimental studies, which eliminate bias due to parental selection into different types of child care, find that, at 36 months, children's cognitive skills are enhanced by high-quality child care, with effect sizes ranging from $d=.12$ in the Early Head Start Study (Love et al., 2005) to $d=.83$ in the Infant Health and Development Program (McCormick et al, 2005) to $d=1.23$ in the Abecedarian study (Campbell, Pungello, MillerJohnson, Burchinal, \& Ramey, 2001). The quality of care in these interventions was likely to be high due to involvement of research staff and use of evidence-based curricula, but child care quality was not actually measured in the treatment or control conditions in the Abecedarian and Infant Health and Development Program interventions.

In the case of younger children (12-24 months), the focus of the present paper, associations between child care quality and cognitive skills are rarely investigated, and were observed in 
a handful of non-experimental studies. These include both small, single-site studies (Burchinal, Roberts, Nabors, \& Bryant, 1996) and larger multisite studies using data collected in the 1990s (NICHD ECCRN, 2006; NICHD ECCRN \& Duncan, 2003). Child samples in prior work were primarily low income (e.g., Early Head Start) or from middleclass backgrounds (e.g., National Institute of Child Health and Development Study of Early Child Care and Youth Development [NICHD-SECCYD]) and thus not fully representative. While studies using the National Longitudinal Study of Youth (Han, Waldfogel, \& BrooksGunn, 2001) are nationally representative, they do not adequately address associations between child care quality and cognitive skills due to the fact that child care quality was not measured. In addition, few large observational studies use statistically rigorous methods to account for parental selection into differing levels of child care quality, types of care, and quantity of care (an exception is work by Dearing, McCartney, and Taylor [2009] with the NICHD-SECCYD sample). The present study therefore uses propensity scores to examine the effect of early child care on cognitive skills at 24 months for children in the ECLS-B, a nationally-representative sample of children born in 2001.

\section{Other Facets of Child Care that May Relate to Cognitive Skills}

In order to account for the relation between child care quality and cognitive skills in an unbiased manner, it is necessary to also account for other facets of early child care that, in prior work, have been linked to children's cognitive skills. Principal among these are the amount of time children spend in child care, the age at which they enter care, and the type of care they receive. Of these, the type of care a child receives (center care or not) may be particularly important because being in center care is associated with increases in cognitive skills (Clarke-Stewart, Gruber, \& Fitzgerald, 1994). While center care predicts cognitive skills over and above the measured quality of care in many studies (Brooks-Gunn, Han, \& Waldfogel, 2002; NICHD ECCRN, 2000b, 2006; NICHD ECCRN \& Duncan, 2003), other research finds no cognitive benefit (measured at age 3 ) for center care versus mother-only care during the first year of a child's life (Han et al., 2001). Loeb, Bridges, Bassok, Fuller, and Rumberger (2007) found that first entering center care between ages 2 and 3 resulted in greater cognitive gains than entering center care in the year prior to kindergarten. Beginning center care prior to two years of age yielded no additional cognitive benefits. Studies that simultaneously examined the amount of time children spent in child care and the quality of child care found only a quality effect on cognitive outcomes (NICHD ECCRN, 2000b; NICHD ECCRN, 2006; NICHD ECCRN \& Duncan, 2003).

Given the above evidence, it is questionable how important these other dimensions of child care are in terms of predicting cognitive skills over and above the measured quality of child care. However, these dimensions of child care may be linked with cognitive skills, and cannot be ignored since doing so may bias any estimates of the effects of child care quality. The present study therefore tests whether these dimensions are related to cognitive skills.

\section{Differential Effects of Child Care on Low-Income Children}

Whether or not child care quality has an overall impact on cognitive skills, it is important to test for the effect of quality on low-income children, since public policies are often targeted toward children from low-income backgrounds. Additionally, estimating the relative magnitudes of effect for low-income and middle-income children is useful for understanding the ability of subsidized, high-quality child care for low-income children to narrow achievement gaps. Some evidence from the preschool period suggests that high-quality care might be a protective factor for low-income children (Burchinal, Roberts, Zeisel, Hennon, \& Hooper, 2006). Quality of care appears to be related to cognitive development for all children (Vandell, 2004), but may be a stronger predictor for low-income children in the 
first year of grade school (Dearing et al., 2009). Although Dearing et al. (2009) considered early care, they did not isolate it from later child care, instead measuring child care quality by the number of times a child experienced high-quality care from 6 to 54 months. It is, thus, important to know whether high-quality infant/toddler care is protective separate from high-quality preschool care.

\section{Present Study}

This study has two aims. First, descriptive information about toddler care experiences are provided using the nationally-representative Early Childhood Longitudinal Survey - Birth Cohort (ECLS-B) to address the need for more information about the type, quantity, and especially quality of child care for toddlers in the US. Second, it addresses the understudied question of whether early cognitive development is related to the quality of toddler care. The ECLS-B provides rich information on children's socio-demographic and psychological characteristics that enable us to use propensity score procedures to reduce bias in estimating the relation between measured child care quality and children's cognitive development from 9 to 24 months of age. We hypothesize that higher quality child care promotes toddlers' cognitive development.

\section{Method}

\section{Participants}

Data are drawn from the Early Childhood Longitudinal Study—Birth Cohort, a nationally representative, longitudinal cohort study of U.S. children born in 2001 (Flanagan \& West, 2005). Sampling from birth certificate records, the ECLS-B oversampled Asian and Pacific Islanders, Native Americans and Alaska Natives, low-birth-weight $(1,500-2,500 \mathrm{~g})$ and verylow-birth-weight (less than 1,500g) children, as well as twins. At 9 and 24 months, children and their caregivers were assessed. The ECLS-B collected data on 10,600 children at the 24month data collection period, representing $99 \%$ of the original study sample. Descriptive statistics on the 24-month sample of children is presented in Table 1, appropriately weighted so that the means, standard deviations, and percentages are representative of children born in the U.S, in 2001. The means and standard deviations in Table 1 were estimated for the maximum number of children in the ECLS-B with valid data.

The $N$ of the full sample of children in Table 1 is larger than the $N$ of children in the main analysis of the paper. For the analytic models assessing child care quality effects on cognitive skills, the sample includes all children with non-missing values for their 24-month child care arrangement, 24 month Bayley mental score, and ECLS-B sample weight (W2C0); and who met eligibility criteria for the child care observation subsample (described below), whether they were in child care or stayed at home with parents $(n=8,350)$. Excluding the 500 children who were ineligible for child care observation, there were some small, but significant differences between children with missing values on these variables and those without. Children with missing values were less likely to be in poverty or speak English at home, had lower 9-month Bayley scores (difference of 0.8), and lower maternal sensitivity (NCATS) scores. These children were more likely to be from non-white, -black, or -Hispanic backgrounds; be the first born child; be born at a very low birth weight; have mothers with less than a high school education and have higher observed HOME scores at 9 months.

\section{Measures}

Comprehensive data were collected on study children when they were approximately 9 and 24 months of age via direct child assessment, parent surveys, and observational measures. 
One-to-one assessments of children's cognitive ability were carried out by ECLS-B staff in the child's home, as were observations of interactions between children and their mothers. Child care observations were also carried out by ECLS-B staff in the settings in which the study child's primary care took place, i.e. where the child spent the most number of hours per week in nonparental care. This was either in a child care center or in the child's, a relative's, or a non-relative's home. Other measures used as control variables were collected via parent surveys, which were administered in children's homes.

All analyses were weighted to provide nationally representative statistics and all tables were created in accordance with IES reporting guidelines. In keeping with NCES/IES reporting guidelines for the ECLS-B, case counts in all tables are rounded to the nearest 50. All descriptive statistics were weighted by the appropriate analytic weight; standard errors were calculated using appropriate PSU and stratum variables supplied by the ECLS-B study.

\section{Socio-demographic, family, and other child and maternal characteristics-}

Detailed demographic information about children and their families was collected via birth certificate records and a 60-minute in-person interview of a child's parent (typically the mother) in which the ECLS-B trained interviewer asked questions aloud and entered the responses in a computer. In order to control for selection into different types of infant and toddler child care experiences (NICHD ECCRN, 2005), our regressions include the following controls: child's age (in months), gender, ethnicity (non-Hispanic white, African American, Hispanic, or other), mother's education (less than HS, HS graduate, some college, BA or greater) and marital status (at 9 months), and whether family income at 9 months was above the $185 \%$ poverty line, the cutoff for Head Start and Free and Reduced Lunch eligibility. In 2003, a family of four below 1.85 times the poverty threshold had an income under $\$ 34,040$. Also accounted for were the following structural features of the family: whether the child was first born, the child's number of older siblings, and whether English was spoken at home. Children's birth weight, either moderately low birth weight (1,500 to 2,500 grams) or very low birth weight (less than 1,500 grams), were included in all models.

Finally, maternal teaching behaviors as measured by the Nursing Child Assessment Teaching Scale (NCATS) were included in all models. When children were 9 months old, the ECLS-B videotaped the parent (the main respondent - typically the mother) selecting an NCATS task that the sampled child had no experience with and then teaching the child that task. Trained raters then coded the videotapes. All models include total parent score from the NCATS $(\alpha=.68)$, which measured parental responsivity to cues (e.g., pausing as child initiates action), cognitive growth fostering (e.g., type of instructions and modeling), socioemotional growth fostering (e.g., cheerleading), and response to distress (e.g., rearranging toys).

Children's cognitive skills-We used the mental scale of the Bayley Short FormResearch Edition (BSF-R) at 24 months as the child outcome. The BSF-R was developed specifically for the ECLS-B and includes a subset of questions from the larger Bayley Scales of Infant Development - Second Edition (BSID-II). At 24 months, the BSF-R mental scale measures a child's performance on tasks requiring memory, problem solving, and language skills. Children were presented tasks such as naming pictures, verbal comprehension, comparing sizes, and matching colors. ECLS-B staffers who administered the BSF-R received a 3-day intensive training on administering and scoring the individual BSF-R items, which culminated in each staffer administering a live BSF-R to a 21 to 30 -month old child to ensure and measure accuracy. Those staffers who ended up administering the BSF-R as part of the full data collection effort scored an average of 93 percent for administration accuracy and an average of 97 percent for scoring accuracy on the BSF-R mental scale. The 
scale scores were calculated using IRT true-score equating so that BSF-R scores were on the same scale as the BSID-II. The observed overall BSF-R mental scale reliability coefficient was 0.975 . ECLS-B guidelines indicate that scale scores are most appropriate for use in analyses of developmental growth over time.

Child care quantity and type-The primary caregiver, typically the mother, was interviewed and asked whether the child was currently in child care, the number of hours per week in child care, and the type of care the child received during the year that the child turned 2-years-old (between January 2003 and December 2003).

Child care quality-For cost reasons, the ECLS-B observed child care quality for a randomly-chosen $25 \%$ subsample of the children whose parents reported using nonparental care for at least 10 hours per week. This group is referred to as the Child Care Observation (CCO) sample. Children who lived in Alaska, Hawaii, resided in American Indian supplement Population Sampling Units, or in a care setting where the language spoken was one other than English or Spanish were not eligible for observation and thus not included in analyses presented below, which amounted to a total of 500 children. All eligible cases where the child lived in poverty and spent the most hours per week in a center-based care arrangement were included in the CCO sample with certainty. All other eligible cases were subsampled at rates designed to reduce the variability in probabilities of selection resulting from the oversampling of low and very low birth weight children in the base ECLS-B sample.

The measures of child care quality in centers and in home-based care used in the ECLS-B were developed to measure comparable dimensions in the two settings from a constructivist theory of early development (Piaget, 2007; Vygotsky, 1978). In this study, the Environmental Rating Scales (ERS), including the Infant/Toddler Environmental Rating Scale (ITERS) and Family Day Care Rating Scale (FDCRS), were used to measure the quality of the child care environment. ERS instruments measure the extent to which adults provide frequent stimulating and sensitive interactions, children have the opportunity to interact with a variety of age-appropriate objects and activities (e.g., water, sand, dress-up clothes), and the child's health and safety is maintained within that environment.

For children in center-based care, ECLS-B observers rated quality of care using the ITERS and for children in family or non-relative home-based care, observers rated child care quality using the FDCRS. The ITERS is a classroom-level measure of child care quality that examines the child's experiences in the care setting. The ITERS examines interactions with adults and peers, exposure to materials and activities, the extent to which and the manner in which routine care needs are met, and the furnishings and displays in the classroom ( 9 month to 2 year User's Manual). Harms, Cryer, and Clifford (1990) report inter-rater and test-retest reliability of $r=0.58$ and $r=0.89$, respectively, internal consistency was $a=$ 0.83 , criterion validity at 83 percent, and content validity between 75 and 86 percent. ECLSB conducted 69 quality control visits to assess reliability of ITERS scoring among its raters, finding an average reliability score across all quality control visits of 87.2 (range 78.3-96.5). The total quality score on the ITERS ( $\alpha=.86$ in the ECLS-B) was used in analyses. This score contains 29 items from the broad categories mentioned above and is on a 7-point scale. The FDCRS is a setting-level measure of child care quality that examines the child's experiences in the care setting, including interactions with adults and peers, materials and activities exposure, whether, how much, and the manner in which routine care needs are met, and classroom furnishings and displays (9-month to 2-year user's manual). Inter-rater reliability for the FDCRS was reported to be $r=.90$, internal consistency was only reported for the subscales and varied from $a=0.7$ to $a=.93$ and while the original manual did not report on concurrent validity, other studies report high correlations $(.80)$ between the 
FDCRS and visitors' ratings of family day care settings (U.S. Administration for Children and Families, 2013). We used the total quality score on the FDCRS ( $\alpha=.88$ in the ECLS-B), which contains 33 items drawn from the categories mentioned above and is also on a 7-point scale.

ECLS-B staffers who rated the quality of care for study children received an extensive multi-day training split roughly 35-65 between direct instruction and field observations that included conducting observations in small groups lead by a trainer and then discussing experiences and scoring with the same small groups in the afternoon. To be certified on either the ITERS or FDCRS, observers were required to have 80 percent of their scores within one point of the consensus score as well as a positive trainer evaluation.

Published guidelines provide an interpretation of the meaning of different values along the 7-point scale of the ITERS and FDCRS: 1 (inadequate), 3 (minimal), 5 (good), and 7 (excellent). Following Kontos, Howes, Shinn, and Galinsky (1995) and Phillipsen, Burchinal, Howes, and Cryer (1997), low-quality care is categorized as a score on the ITERS or FDCRS below 3, medium-quality care as a score greater than or equal to 3 and less than 5, and high-quality care as a score of 5 and above. In using this approach to measure overall child care quality, we borrow from prior research (Burchinal et al., 2000) that treated scores from different child care quality measures equally in analyses that accounted for potential differences in mean scores and variability across the two instruments. To ensure the quality construct is appropriate, the regression models described below allow for the combined quality score to vary by type of care (by including a type-ofcare-by-quality interaction term) to account for any differences that might be measure based. A series of robustness checks, in which the final model is estimated separately by child care type, among others, are estimated to ensure the validity of treating ITERS and FDCRS scores as equivalent. The outcomes of these checks are presented at the end of the Results section.

Child care categorization for analysis-Two issues complicate ECLS-B-based analyses of child care type and quality. First, type of care has been divided into exclusive parental care and routine nonparental care. It is noteworthy that the nonparental group is further distinguished by where child care is provided and who provides it (i.e., relatives, non-relatives in a home-based setting, or center care). Second, as mentioned above, child care quality was only observed for a random subsample of about $25 \%$ of children who were in at least 10 hours of nonparental care, coded here as receiving high-, medium-, or lowquality care based on their ITERS or FDCRS scores. Thus, for analytic purposes, a total of five child care categories were possible, which encompassed all children in the ECLS-B at 24 months: i) exclusive parental care; ii) nonparental care that was not-observed; iii) low quality care; iv) medium quality care; and v) high quality care.

\section{Analytic Approach}

Two sets of analyses were conducted. The first provides a nationally representative description of the child care experiences of 2-year-olds in 2003. The type, quantity, and quality of care are described for the entire sample and separately by poverty status of the family. The use of appropriate ECLS-B sample weights ensures that the child care experiences described are nationally representative

The second set of analyses estimated differences in cognitive development among children in high-, medium-, and low-quality toddler care. Preliminary analyses tested quality-byquantity and quality-by-type interactions and found no consistent evidence of them. Based on the results from this analysis, the analyses presented in this paper utilize propensity score methodology to test for associations between child care quality and 24 month Bayley mental 
scores across the entire ECLS-B sample, including those children in exclusive parental care or for whom child care was unobserved.

\section{Generalized propensity scores to account for selection into child care-To}

help account for the selection processes involved in the decision to keep a child at home or place him/her in child care (whether low, medium, or high in quality), generalized propensity score models were estimated on the entire ECLS-B sample. Because the ECLS-B is an observational study, estimated associations between child care quality and child outcomes may over- or understate causal effects owing to family and child factors that are correlated with child care quality and child outcomes. In cases when there are a large number of such factors, and the distribution of these factors varies with treatment status (child care quality in this analysis), covariate adjustment via regression analysis alone is often inadequate (Imbens, 2000). Building on Rosenbaum and Rubin's (1983) propensity score adjustment technique, Imbens (2000) and Imbens and Wooldridge (2009) outline a method of adjusting for treatment choice based on pre-treatment characteristics in situations where treatment takes on more than two values, termed generalized propensity scores.

First, a multinomial logistic regression is estimated to predict whether a child was in parental care, nonparental care not observed, low quality, medium quality, or high quality nonparental care during the 12-24 month period using a wide range of covariates. Covariates drawn from the literature include the child's development, ethnicity, and poverty status, were related to family choice of child care (Early \& Burchinal, 2001; Gamble, Ewing, \& Wilhelm, 2009). In addition, the representativeness of the ECLS-B sample provides further assurances that the analytic models to predict propensity to select care are reliable (Burchinal \& Nelson, 2000). A list of the variables used in the propensity score analysis, including descriptive statistics for each of the child care groups, and the results of the multinomial logistic regression are presented as online supplementary tables 1 and 2, respectively. The coefficient estimates from the multinomial logistic regression predicting care categories are then used to calculate a child's probability of being in each one of the different categories. The generalized propensity score for each child is equal to the inverse of the child's probability of being in the appropriate care category given their 24-month parenting or child care experience.

We assessed the adequacy of the propensity score model by checking for balance across the treatment groups on each of the key independent variables in our final model, both before and after propensity score adjustment. Before propensity score adjustment, only 3 of the 17 independent variables in our final model were balanced across treatment groups; after propensity score adjustment, balance across treatment groups was achieved in 11 of the 17 independent variables. In addition, for each of the 17 variables, we conducted 10 pairwise comparisons of standardized means for a total of 170 comparisons. Only $13(8 \%)$ of the propensity-adjusted comparisons had standardized mean differences below -.1 or above .1 , a common criteria for assessing balance. By comparison, 64 of the $170(38 \%)$ of the prepropensity score adjusted comparisons had standardized mean differences outside the -.1 to .1 interval.

The final step in the analysis is to use the generalized propensity scores to weight an OLS regression predicting the Bayley cognitive score at 24 months using indicators for child care type and the set of variables in Table 2 as independent predictors as follows, 


\author{
(24 month cognitive score $)_{i}=\beta_{0}+\beta_{1}(9 \text { month cognitive score })_{i}+\beta_{2}$ (whether \\ low quality care $)_{i}+\beta_{3}$ (whether high quality care $)_{i}+\beta_{4}$ (whether parental care $)_{i}$ \\ $+\beta_{5}$ (whether nonparental care not observed $)_{i}+\beta_{6}$ (whether not low income $)_{i}+\beta_{7}($ controls $)+\varepsilon_{i}$.
}

By including the dummy variables in the equation for children in parental and unobserved nonparental care, a full sample was maintained and regression coefficients equal the difference in average outcomes for these groups of children as compared with the group with observed, medium-quality child care. This generalized propensity score model should account for potential selection bias to the variables included in the multinomial regression models, thereby reducing bias in the estimation of the toddler quality effects.

\title{
Results \\ Child Care Experiences for 24-Month-Old Toddlers
}

The first set of analyses described the child care experiences at 2 years for the nationally representative sample, ECLS-B. Table 2 provides nationally representative estimates of the type, quantity, and quality of care as experienced by children who were 24 months of age in 2003 , both for the entire sample and separately by poverty status.

Type and quantity of toddler care-About half (51\%) of two-year olds were in parental care (experienced less than 10 hours per week of nonparental care); $18 \%$ were in relative care; $15 \%$ were in child care homes; and $16 \%$ of children were in child care centers. Significantly higher proportions of low-income children were in parental care (odds ratio, 1.69) and relative care (odds ratio, 1.22) relative to middle- and upper- income age mates, while the opposite was true for enrollment in center care (odds ratio .58). Table 2 further breaks out the average amount of time spent by toddlers in more than 10 hours per week of nonparental care across various types of care arrangements. Children in either relative care or child care homes spent an average of 32 hours in those settings, while children who were primarily in center care spent an average of 34 hours per week there. Center care was the only setting with a statistically significant difference between the amount of time spent by low income ( $M=32$ hours/week) and middle- or upper-income children ( $M=37$ hours/ week), $F(1,1350)=19.02, p<.001$.

Quality of toddler care-Of those 24-month-olds in nonparental care settings, mediumquality care was experienced by the largest percentage of children $(61 \%)$ followed by lowquality care (26\%) and high-quality care (13\%). Low-income children, compared with medium-and upper-income children, were more often in low-quality care $(43 \% \mathrm{v} 16 \%)$ and less often in medium- (48\% v 69\%) or high-quality care (9\% v 15\%), $\chi^{2}=38.17, \mathrm{p}<.001$. Whereas mean child care quality scores were equivalent for low- and medium-income children in child care centers, low-income children had significantly lower mean quality scores in either relative care and child care homes $F(1,800)=119.96, p<.001$.

\section{Child Care Quality Impacts on 24 Month Cognitive Skills}

To estimate toddler care quality effects on 24 month cognitive skills, the presentation of results begins with the children whose child care settings were observed and rated by ECLSB staffers, testing whether the quality, quantity, and type of child care are related to cognitive skills at 24 months. Then a multinomial propensity score model was estimated with children in medium-quality care for 35 hours or less as the reference cell and weights calculated for the following groups: low quality ( $<=35$ hours or $>35$ hours), medium quality ( $>35$ hours), and high quality ( $<=35$ hours or $>35$ hours). Results in Table 3 show effect size 
estimates from regressions of 24 month Bayley mental scores on joint indicators of quality and quantity of care, whether in center care or not, income status, and controls. Results in column 1 come from a regression weighted by the appropriate ECLS-B child care observation sampling weight, while the regression results in column 2 are propensity score weighted, specifically the inverse of the child's propensity to be in the quantity by quality categories described above. Post-hoc tests of the main effects of child care quality, quantity, and type reveal that only the main effect of quality was statistically significant, and that the quality-by-quantity interaction was not statistically significant. Follow-up analyses (not shown) also indicated that quality-by-poverty status interactions were not statistically significant (all $p$-values $>0.15$ ). Child care quality appears to be the only child care dimension significantly associated with cognitive skills at 24 months.

The above analysis included only children in child care and therefore does not take into account potential bias associated with who decides to use or not use toddler care. This decision involves two steps: 1) families choose whether or not to put their child in child care and 2) if they opt into child care, families decide to place their child into the best care they can given a wide variety of constraints. In reality, this means a child can get placed into a high-, medium-, or low-quality setting. To help account for these selection processes and achieve less biased estimates of the association between child care quality and cognitive skills, we analyzed data from the entire ECLS-B sample. These analyses employed indicator variables for each of the following: whether a child experienced exclusive parental care, whether a child was in nonparental care of unobserved quality, and whether a child was observed to be in low-, medium-, or high-quality child care.

To address this question, three sets of analyses were conducted. The first column in Table 4 shows the results from a multiple regression (i.e., OLS) analyses. The second column shows the results from multiple regression analyses that included the appropriate sample weights to make the results nationally representative for 2 -year-olds in 2003 . The third column shows the results from multiple regression analyses that included the propensity score weights. In all analyses, the coefficients for child care type compares that group with the group of children in middle quality toddler care adjusted for the same covariates. The propensity score approach (column 3 ) is the preferred method of dealing with the selection processes involved in early child care decisions, and is the focus of the rest of the results section. All continuous variables, independent and dependent, were standardized to have a mean of zero and standard deviation of 1 so coefficients can be interpreted in terms of effect sizes.

The propensity-score-weighted estimates in column 3 indicate that low-quality care is associated with significantly lower Bayley scores than medium-quality care $(d=-0.17)$, and high-quality care yields significantly higher Bayley scores than medium-quality care $(d=$ 0.38 ). The association between high-quality care and Bayley scores is more than twice as large of an effect size compared with the negative effect size associated with low-quality care. The Bayley scores of children in either parental care or home-based (unobserved) child care are not reliably different from the scores of children in medium-quality settings. Though not shown, tests for interactions between poverty status and child care quality indicated that the effect of higher-quality child care does not vary by poverty status. Likewise, the negative cognitive skills effect of being in low-quality care was not reliably different by poverty status.

\section{Robustness of Results}

The models in Table 4 group ITERS and FDCRS scores into categories based on the assumption that the scores from these two measures are equivalent. Descriptively, FDCRS scores for children in the child care sample tend to be clustered around the lower-end of the scale while ITERS scores are more clustered at the upper end of the scale. We tested the 
equivalence assumption by running separate regression models for children in center care (ITERS) and children in home-based care (FDCRS-results available from first author). In home-based care settings, a significant increase in Bayley scores is observed only for children in medium-quality care (versus low-quality care). In center care settings, Bayley scores are significantly higher only for children in high-quality care (versus medium-quality care). In models where the quality scores are kept as continuous variables, coefficients for the effect of child care quality on Bayley scores are both significantly positive and of a similar magnitude. Finally, we ran a model identical to the final model in Table 4 that included an indicator for whether childcare was assessed with the ITERS or FDCRS, to allow for the possibility of an instrument-based effect. Results from this model did not change the significance or direction of the key treatment variables, nor was quality assessment by ITERS/FDCRS associated with 24 month Bayley scores. We conclude that our results are, in general, robust to the assumption of equivalence between the ITERS and FDCRS.

\section{Discussion}

In this paper, we employed a nationally representative sample to examine the distribution of the quality of toddler care and its relation to cognitive performance at 24 months of age. As with prior work with preschool-age children, the results demonstrate that nonparent care is now normative for a large fraction of U.S. toddlers, and that child care quality is related to early cognitive development as well as to preschool language, academic, and social development.

The descriptive analyses of the children's child care indicated that almost half of 2-year-old children in 2003 experienced routine nonparental care, which is consistent with estimates from non-representative samples (NICHD ECCERN, 2006) as well as from earlier representative samples such as the National Survey of American Families and the National Household Education Surveys Program. For those children in child care, about one-third were cared for by relatives, another third by nonrelatives in home-based care, and the last third in child care centers. Toddlers in child care tended to experience between 30 and 35 hours of care per week. The quality of center care was, on average, in the medium range according the ITERS $(M=4.2)$ and in the low-medium range according to the FDCRS ( $M=$ 3.5 ), with $60 \%$ of toddlers attending a setting rated in the medium range. Consistent with prior reports (NICHD ECCRN, 2006), toddlers from low-income families were much less likely to be in child care, and if in care, experienced lower quality care on average in homebased but not center-based settings.

These findings provide a consistent, but more complete, picture of the child care experiences of toddlers in the US. As in the Cost, Quality, and Outcomes Study (Helburn, 1995), they suggest that the quality of toddler care tends to be somewhat lower than the quality of preschool care. This is not surprising as it is more expensive to provide high-quality infant/ toddler care than preschool care (Helburn \& Howes, 1996) given that infants and toddlers require more adult caregiving for routine care and more individual interactions to scaffold early learning and social development. Unlike the NICHD Study of Early Child Care (NICHD ECCRN, 2002), these findings suggest that quality of home-based care was lower than the quality of center-based care for 2-year-olds. This discrepancy is likely related to differences in the samples and the quality measures. The NICHD study was not nationally representative, although both are cohort studies of children, albeit separated in time by 10 years.

These results could be helpful to policy makers as they wrestle with questions of how best to help parents obtain child care for toddlers and create tracking mechanisms to promote higher 
quality care (e.g., quality rating and improvement systems; Tout et al., 2010). Existing state rating systems include some measure of the quality of infant/toddler care provided; however, these are typically combined with the quality of preschool care in settings that provide care for infants and preschoolers. It might be more helpful for parents if the quality of infant/ toddler care was rated separately from the quality of the preschool care. This study also extends our understanding of toddler care by demonstrating associations between child care quality and cognitive skills at 24 months using a nationally representative sample. Compared with low-quality care, children had modestly to moderately higher cognitive scores in medium-quality care $(d=.17)$ and high-quality care $(d=.38)$. These findings are generally consistent with those reported in previous studies with non-representative samples (Burchinal et al., 1996; Clarke-Stewart et al., 1994; NICHD ECCRN \& Duncan, 2003; Schliecker, White, \& Jacobs, 1991), and provide similar or somewhat larger effect sizes. Furthermore, in models where child care quality, type and amount of time spent in care were considered, no evidence emerged that the type or amount of child care independently predicted cognitive skills at 24 months over and above child care quality. These findings provide further evidence that early cognitive skills are enhanced in higher-quality care settings, even for children as young as two years of age.

Publicly-funded preschool programs are designed to help address poverty-based achievement gaps, largely built on the assumption that exposure to high-quality care will improve school readiness skills for children from low-income families. This study provides some information that can aid policymakers as they consider whether such policies might help prevent the income achievement gap that is apparent by two years of age in the ECLSB. Differences in cognitive scores by poverty status were small but statistically significant at 9 months of age, but grew substantially to approximately half a standard deviation at 24 months of age. High-quality child care at two years of age appears to convey similar benefits across the income specturm; we could not reject the hypothesis of equal impacts by poverty status. However, since fewer low-income children were in medium- and high-quality care, increasing the proportion of low-income toddlers in medium- and high-quality care may help reduce the income achievement gap.

A first potential limitation of this study is that overall scores from two different measures of child care quality were treated as equivalent. While the ITERS and FDCRS share much in common, from the standpoint of their development as measures of child care quality, it is unclear the degree to which scores from the separate measures can be considered equivalent. To address this limitation, we ran a series of regression models for each type of care separately, which supported the results from models that treated the scores as equivalent. A second limitation of this study is that while propensity score methods account for selection on observed variables, the possibility of selection bias due to unobserved confounds still exists. For this reason, it is important to be careful in interpreting the practical implications of this research, as the findings are not causal. Future studies might replicate and build on the present analysis by focusing on non-cognitive outcomes. The Bayley measure of cognitive development is highly reliable and valid but only measures one aspect of the developing child. Ideally, we would be able to use other reliable and valid measures of children's behavioral outcomes. Such variables were recorded by the staffer administering the 24-month Bayley, and have been successfully analyzed by others (Morgan, Farkas, Hillemeier, and Maczuga, 2009).

Much of the research on whether the quality of early child care experiences matter for children's cognitive skills development have focused on the pre-K period. The present study addressed this issue for 12-24-month-old children, and found that both medium- and highquality child care are positively associated with the cognitive skills development of all children. No evidence was found to indicate that higher-quality child care was more 
beneficial for low-income children than non-low-income children. However, the fact that low-income children were much less likely to experience medium- or high-quality care at 24 months of age suggest that child care quality may play a role in widening the achievement gap between infancy and the preschool years. Public funding that increases the supply of high-quality care for low-income children might therefore help narrow the cognitive skills gap at two years of age.

\section{Supplementary Material}

Refer to Web version on PubMed Central for supplementary material.

\section{Acknowledgments}

We are grateful to the Institute of Education Sciences (IES) for supporting this work through grant R305A090467-10 awarded to Principal Investigator George Farkas and Co-Principal Investigators Margaret Burchinal and Greg J. Duncan. The opinions expressed are those of the authors and do not represent views of the Institute or the U.S. Department of Education. Research reported in this publication was also supported by the Eunice Kennedy Shriver National Institute of Child Health \& Human Development of the National Institutes of Health under Award Number P01HD065704. The content is solely the responsibility of the authors and does not necessarily represent the official views of IES, the U.S. Department of Education, or the National Institutes of Health.

\section{References}

Bodrova, E.; Leong, D. Vygotskian perspectives on teaching and learning early literacy. In: Dickinson, DK.; Neuman, SB., editors. Handbook of early literacy research. New York, NY: Guilford; 2006.

Bronfenbrenner, U.; Morris, PA. The bioecological model of human development. In: Damon, W.; Lerner, RM., editors. Handbook of child psychology, Vol. 1: Theoretical models of human development. 6. New York, NY: Wiley; 2006. p. 793-828.

Brooks-Gunn J, Han W, Waldfogel J. Maternal employment and child cognitive outcomes in the first three years of life: The NICHD Study of Early Child Care. Child Development. 2002; 73(4):10521072.10.1111/1467-8624.00457 [PubMed: 12146733]

Burchinal MR, Cryer D. Diversity, child care quality, and developmental outcomes. Early Childhood Research Quarterly. 2003; 18:401-426.10.1016/j.ecresq.2003.09.003

Burchinal, M.; Kainz, K.; Cai, Y. How well do our measures of quality predict child outcomes? A meta-analysis and coordinated analysis of data from large-scale studies of early childhood settings. In: Zaslow, M., editor. Quality measurement in early childhood settings. Baltimore, MD: Paul H. Brookes; 2011.

Burchinal MR, Nelson L. Family selection and child care experiences: implications for studies of child outcomes. Early Childhood Research Quarterly. 2000; 15(3):385-411.10.1016/ S0885-2006(00)00072-7

Burchinal MR, Roberts JE, Nabors LA, Bryant DM. Quality of center child care and infant cognitive and language development. Child Development. 1996; 67(2):606-620.10.2307/1131835 [PubMed: 8625731]

Burchinal M, Roberts JE, Zeisel SA, Hennon EA, Hooper S. Social risk and protective child, parenting, and child care factors in early elementary school years. Parenting: Science and Practice. 2006; 6:79-113.10.1207/s15327922par0601_4

Camilli G, Vargas S, Ryan S, Barnett WS. Meta-analysis of the effects of early education interventions on cognitive and social development. Teachers College Record. 2010; 112(3):579-620.

Campbell FA, Pungello EP, Miller-Johnson S, Burchinal MR, Ramey C. The development of cognitive and academic abilities: Growth curves from an early intervention educational experiment. Developmental Psychology. 2001; 37(2):231-242.10.1037/0012-1649.37.2.231 [PubMed: 11269391]

Clarke-Stewart, A.; Gruber, CP.; Fitzgerald, LM. Children at home and in day care. Hillsdale, NJ: Erlbaum; 1994. 
Dearing E, McCartney K, Taylor BA. Does higher quality early child care promote low-income children's math and reading achievement in middle childhood? Child Development. 2009; 80(5): 1329-1349.10.1111/j.1467-8624.2009.01336.x [PubMed: 19765003]

Early DM, Burchinal MR. Early childhood care: relations with family characteristics and preferred care characteristics. Early Childhood Research Quarterly. 2001; 16(4):475-497.10.1016/ S0885-2006(01)00120-X

Flanagan KD, West J. Children born in 2001: First results from the base year of the Early Childhood Longitudinal Study, Birth Cohort (ECLS-B). Education Statistics Quarterly. 2005; 6(4)

Gamble WC, Ewing AR, Wilhelm MS. Parental perceptions of characteristics of nonparental child care: Belief dimensions, family, and child correlates. Journal of Child and Family Studies. 2008; 18(1):70-82.10.1007/s10826-008-9208-z

Gormley WT, Gayer T, Phillips D, Dawson B. The effects of universal pre-K on cognitive development. Developmental Psychology. 2005; 41(6):872-884.10.1037/0012-1649.41.6.872 [PubMed: 16351334]

Han W, Waldfogel J, Brooks-Gunn J. The effects of early maternal employment on later cognitive and behavioral outcomes. Journal of Marriage and Family. 2001; 63(2):336-354.10.1111/j. 1741-3737.2001.00336.x

Harms, T.; Cryer, D.; Clifford, RM. Infant/toddler Environment Rating Scale. New York, NY: Teachers College Press; 1990.

Helburn, S., editor. Cost, quality, and child outcomes in child care centers: Technical Report. Denver: Department of Economics, Center for Research in Economic and Social Policy, University of Colorado at Denver; 1995.

Helburn SW, Howes C. Child care cost and quality. The Future of Children. 1996; 6(2):62-82. [PubMed: 8972128]

Howes C, Burchinal M, Pianta R, Bryant D, Early DM, Clifford R. Ready to learn? Children's preacademic achievement in pre-kindergarten programs. Early Childhood Research Quarterly. 2008; 23:27-50.10.1016/j.ecresq.2007.05.002

Imbens $\mathrm{G}$. The role of propensity score in estimating dose-response in observational studies for causal effect. Biometrika. 2000; 3:706-710.10.1093/biomet/87.3.706

Imbens GW, Wooldridge JM. Recent developments in the econometrics of program evaluation. Journal of Economic Literature. 2009; 47(1):5-86.10.1257/jel.47.1.5

Kontos S, Howes C, Galinsky E. Does training make a difference to quality in family child care? Early Childhood Research Quarterly. 1996; 11(4):427-445.10.1016/S0885-2006(96)90016-2

Laughlin, L. Current Population Reports. Washington, DC: U.S. Census Bureau; 2010. Who's minding the kids?: Child care arrangements, Spring 2005/Summer 2006; p. 70-121.Retrieved from: http:// www.census.gov/prod/2010pubs/p70-121.pdf

Loeb S, Bridges M, Bassok D, Fuller B, Rumberger RW. How much is too much? The influence of preschool centers on children's social and cognitive development. Economics of Education Review. 2007; 26(1):52-66.10.1016/j.econedurev.2005.11.005

Love JM, Kisker EE, Ross C, Raikes H, Constantine J, Boller K, Vogel C. The Effectiveness of Early Head Start for 3-Year-Old Children and Their Parents: Lessons for Policy and Programs. Developmental Psychology. 2005; 41(6):885-901.10.1037/0012-1649.41.6.885 [PubMed: 16351335]

Mashburn AJ, Pianta RC, Hamre BK, Downer JT, Barbarin OA, Bryant D, Howes C. Measures of classroom quality in prekindergarten and children's development of academic, language, and social skills. Child Development. 2008; 79(3):732-749.10.1111/j.1467-8624.2008.01154.x [PubMed: 18489424]

McCormick M, Brooks-Gunn J, Buka S, Goldman J, Yu J, Salganik M, Patrick H. Early intervention in low birth weight premature infants: Results at 18 years of age for the Infant Health and Development Program. Pediatrics. 2006; 117:771-780.10.1542/peds.2005-1316 [PubMed: 16510657]

Morgan PL, Farkas G, Hillemeier MM, Maczuga S. Risk factors for learning-related behavior problems at 24 months of age: population-based estimates. Journal of abnormal child psychology. 2009; 37(3):401-413.10.1007/s10802-008-9279-8 [PubMed: 19057886] 
NICHD Early Child Care Research Network. The relation of child care to cognitive and language development. Child Development. 2000a; 71:960-980.10.2307/1132337 [PubMed: 11016559]

NICHD Early Child Care Research Network. Characteristics and quality of child care for toddlers and preschoolers. Applied Developmental Science. 2000b; 4(3):116-35.10.1207/ S1532480XADS0403_2

NICHD Early Child Care Research Network. Early child care and children's development prior to school entry: Results from the NICHD Study of Early Child Care. American Educational Research Journal. 2002; 39:133-164.10.2307/3202474

NICHD Early Child Care Research Network. Early child care and children's development in the primary grades: Follow-up results from the NICHD Study of Early Child care. American Educational Research Journal. 2005; 42(3):537-570.10.2307/3700462

NICHD Early Child Care Research Network. Child-care effect sizes for the NICHD study of early child care and youth development. American Psychologist. 2006; 61(2):99116.10.1037/0003-066X.61.2.99 [PubMed: 16478355]

Duncan G. NICHD Early Child Care Research Network. Modeling the impacts of child care quality on children's preschool cognitive development. Child Development. 2003; 74(5):14541475.10.1111/1467-8624.00617 [PubMed: 14552408]

Peisner-Feinberg E, Burchinal M. Relations between preschool children's child-care experiences and concurrent development: The cost, quality, and outcomes study. Merrill-Palmer Quarterly. 1997; 43:451-477.

Peisner-Feinberg ES, Burchinal MR, Clifford RM, Culkin ML, Howes C, Kagan SL, Yazejian N. The relation of preschool childcare quality to children's cognitive and social developmental trajectories through second grade. Child Development. 2001; 72(5):1534-1553.10.1111/1467-8624.00364 [PubMed: 11699686]

Phillipsen L, Burchinal MR, Howes C, Cryer D. The prediction of process quality from structural features of child care. Early Childhood Research Quarterly. 1997; 12(3):281-303.10.1016/ S0885-2006(97)90004-1

Piaget, J. The Child's Conception of the World. Lanham, MD: Rowman \& Littlefield; 2007.

Pianta RC, Barnett WS, Burchinal M, Thornburg KR. The effects of preschool education: What we know, how public policy is or is not aligned with the evidence base, and what we need to know. Psychological Science in the Public Interest. 2009; 10(2):49-88.10.1177/1529100610381908

Reynolds AJ, Temple JA, Robertson DL, Mann EA. Age 21 cost-benefit analysis of the Title I Chicago child-parent centers. Educational Evaluation and Policy Analysis. 2002; 24(4):267303.10.3102/01623737024004267

Rosenbaum PR, Rubin DB. The central role of the propensity score in observational studies for causal effects. Biometrika. 1983; 70(1):41-55.10.1093/biomet/70.1.41

Rutter M. ERA Study Team. Developmental catch-up, and deficit, following adoption after severe global early privation. The Journal of Child Psychology and Psychiatry and Allied Disciplines. 1998; 39(4):465-476.10.1111/1469-7610.00343

Schliecker E, White DR, Jacobs E. The role of day care quality in the prediction of children's vocabulary. Canadian Journal of Behavioural Science/Revue canadienne des sciences du comportement. 1991; 23(1):12.10.1037/h0078960

Tout, K.; Starr, R.; Soli, M.; Moodie, S.; Kirby, G.; Boller, K. The Child Care Quality Rating System (QRS) assessment: Compendium of quality rating systems and evaluations. Washington, DC: Child Trends; 2010. Retrieved from http://www.acf.hhs.gov/sites/default/files/opre/ qrs_compendium_final.pdf

U.S. Administration for Children and Families, Office of Planning, Research \& Evaluation. Family Child Care Environment Rating Scale-Revised Edition (FCCERS-R), 2007. 2013. Retrieved from http://archive.acf.hhs.gov/programs/opre/ehs/perf_measures/reports/resources_measuring/ res_meas_impk.html

Vandell D. Early child care: The known and the unknown. Merrill-Palmer Quarterly. 2004; 50(3):387414. 
Votruba-Drzal E, Coley RL, Chase-Lansdale PL. Child care and low-income children's development: Direct and moderated effects. Child Development. 2004; 75(1):296-312.10.1111/j. 1467-8624.2004.00670.x [PubMed: 15015691]

Vygotsky, L. Mind in Society. Cambridge, MA: Harvard University Press; 1978. 


\section{Highlights}

- The quantity, type, and quality of child care are reported on for U.S. toddlers in 2003.

- Low income children were in lower quality care than non-low income children.

- Propensity score adjustment is used to account for parental selection into child care.

- Quality of child care is significantly associated with cognitive skills at 24months.

- Increasing quality of care for low income children critical to closing cognitive skills gap. 
Table 1

Family and Child Characteristics ( $\mathrm{N}=10200$, unless otherwise noted)

\begin{tabular}{|c|c|}
\hline Variable & Mean $(S D)$ or Percentage \\
\hline \multicolumn{2}{|l|}{$\underline{\text { Cognitive Development }}$} \\
\hline Bayley Mental Score 9 months & $76.69(9.79)$ \\
\hline 9-month Low Income/Non-low Income Difference in Bayley Scores & $\begin{array}{l}.63^{* *} \\
F(1,10200)=10.51\end{array}$ \\
\hline Bayley Mental Score 24 months $(N=8900)$ & $127.09(10.65)$ \\
\hline 24-month Low Income/Non-low Income Difference in Bayley Scores & $\begin{array}{l}5.02^{* * *} \\
F(1,8900)=525.30\end{array}$ \\
\hline \multicolumn{2}{|l|}{$\underline{\text { Child and Family Characteristics }}$} \\
\hline Family Income ( $>1.85$ poverty threshold) & $51.51 \%$ \\
\hline English Spoken at Home & $81.17 \%$ \\
\hline Parent-reported Home Score & $12.30(2.45)$ \\
\hline ECLS-B Observed HOME Score & $10.58(1.28)$ \\
\hline Single Mom & $19.57 \%$ \\
\hline Maternal Sensitivity (NCATS) & $34.64(4.17)$ \\
\hline \multicolumn{2}{|l|}{ Maternal Education $(N=8950)$} \\
\hline Less than High School Diploma & $17.78 \%$ \\
\hline High School Diploma $^{a}$ & $30.94 \%$ \\
\hline Some College & $26.57 \%$ \\
\hline Bachelor's Degree or Higher & $24.71 \%$ \\
\hline \multicolumn{2}{|l|}{ Child Characteristics } \\
\hline Black & $13.77 \%$ \\
\hline Hispanic & $25.58 \%$ \\
\hline White $^{a}$ & $53.38 \%$ \\
\hline Other & $7.27 \%$ \\
\hline Male & $51.11 \%$ \\
\hline Child's Age in Months at 9 Month Data Collection & $10.48(1.95)$ \\
\hline Hours of Care at 9 Months & $15.61(20.03)$ \\
\hline Maternal Care at 9 Months & $49.92 \%$ \\
\hline First-born Status & $40.71 \%$ \\
\hline Number of Siblings & $.99(1.11)$ \\
\hline Low Birth Weight & $6.21 \%$ \\
\hline Very Low Birth Weight & $1.29 \%$ \\
\hline \multicolumn{2}{|l|}{ Note. } \\
\hline $\begin{array}{l}\begin{array}{l}a \\
\text { Omitted category in multinomial logistic regression. }\end{array} \\
p<0.05\end{array}$ & \\
\hline$* * x<0.01$ & \\
\hline$* * * * 0.001$ & \\
\hline
\end{tabular}


All variables were measured at 9 months unless otherwise noted. Means were weighted using ECLS-B weight W1C0 for 9 month variables and W2 $\mathrm{C} 0$ for 24-month Bayley score and maternal education. Ns are rounded to the nearest 50. 
Table 3

Child Care Sample Regressions Predicting Bayley Mental Scale at 24 Mos. (effect size estimates)

\begin{tabular}{|c|c|c|}
\hline & $\begin{array}{c}\text { (1) Child Care Sample Weight } \\
\qquad B(S E)\end{array}$ & $\begin{array}{c}\text { (2) Propensity Weight } \\
B(S E)\end{array}$ \\
\hline Low Quality <=35 Hours & $-0.25^{*}(0.10)$ & $-0.10(0.09)$ \\
\hline Low Quality >35 Hours & $-0.27^{* *}(0.10)$ & $-0.19^{*}(0.09)$ \\
\hline Medium Quality <=35 Hours & Reference & Reference \\
\hline Medium Quality >35 Hours & $0.14(0.08)$ & $0.15^{*}(0.08)$ \\
\hline High Quality <=35 Hours & $0.08(0.12)$ & $0.24(0.14)$ \\
\hline High Quality >35 Hours & $0.08(0.12)$ & $0.33^{* *}(0.11)$ \\
\hline Center Care 24 Mos. & $-0.19(0.07)$ & $0.04(0.07)$ \\
\hline Medium Income & $0.04(0.08)$ & $-0.03(0.09)$ \\
\hline \multicolumn{3}{|l|}{$\underline{\text { Main effect of quality tests }}$} \\
\hline Low vs. medium & $-3.93^{* * *}(0.89)$ & $-3.26^{* * *}(0.78)$ \\
\hline Medium vs. high & $0.14(0.94)$ & $2.29 *(0.96)$ \\
\hline Low vs. high & $-3.47^{* *}(1.18)$ & $-4.71^{* * *}(1.07)$ \\
\hline Main effect of quantity & $-0.38(0.72)$ & $-0.53(0.71)$ \\
\hline Quality X Quantity Interactions & ns & ns \\
\hline Constant & $-0.07^{* * *}(0.16)$ & $-0.02^{* * *}(0.17)$ \\
\hline$N$ & 1350 & 1350 \\
\hline$R^{2}$ & 0.26 & 0.29 \\
\hline $\begin{array}{l}\text { Note. } \\
*{ }^{*}<<0.05\end{array}$ & & \\
\hline ** $p<0.01$ & & \\
\hline${ }^{* * * *} p<0.001$. & & \\
\hline
\end{tabular}

Regression 1 was weighted using the 24 month child care weight $W 2 C 2 P 0$ and standard errors were calculated using the appropriate PSU and stratum variables. Regression 2 was weighted by the inverse of the propensity that a child would be in the actual care category they were observed to be in. Controls include 9-month Bayley, child's ethnicity, gender, and age, Mother's education, maternal sensitivity (NCATS), family structure (whether single mother), whether low or very low birth weight, whether home language is English, whether first born, and number of siblings. Ns were rounded to the nearest 50 . 
Table 4

Propensity Score Regressions Predicting Bayley Cognitive Score at 24 Months by Quality and Type of Child Care (effect size estimates)

\begin{tabular}{|c|c|c|c|}
\hline & $\begin{array}{c}\text { (1) Unweighted } \\
\beta \text { (SE) }\end{array}$ & (2) ECLS-B Wave 2 Weight & (3) Propensity Score Weighted \\
\hline \multicolumn{4}{|l|}{ Toddler Care Groups } \\
\hline Low quality care & $-0.25^{* * *}(0.06)$ & $-0.29^{* * *}(0.06)$ & $-0.17^{*}(0.08)$ \\
\hline Medium quality care & Reference & Reference & Reference \\
\hline High quality care & $0.13(0.07)$ & $0.07(0.08)$ & $0.38^{* * *}(0.11)$ \\
\hline Exclusive parental care & $-0.16^{* * *}(0.04)$ & $-0.17^{* * *}(0.04)$ & $-0.05(0.06)$ \\
\hline In child care - quality not observed & $-0.11^{* *}(0.04)$ & $-0.16^{* * *}(0.04)$ & $0.01(0.06)$ \\
\hline \multicolumn{4}{|l|}{ Child Covariates } \\
\hline Above 1.85 poverty threshold & $0.09^{* * *}(0.02)$ & $0.10^{* *}(0.03)$ & $0.01(0.06)$ \\
\hline $9 \mathrm{~m}$ cognitive score & $0.32^{* * *}(0.02)$ & $0.32^{* * *}(0.03)$ & $0.32^{* * *}(0.04)$ \\
\hline \multicolumn{4}{|l|}{ Race/ethnciiy (reference: white) } \\
\hline Black & $-0.29^{* * *}(0.03)$ & $-0.31^{* * *}(0.04)$ & $-0.26^{* * *}(0.07)$ \\
\hline Hispanic & $-0.23^{* * *}(0.03)$ & $-0.23^{* * *}(0.04)$ & $-0.12(0.08)$ \\
\hline Other & $-0.07^{* *}(0.03)$ & $-0.14^{* * *}(0.04)$ & $0.03(0.08)$ \\
\hline Male & $-0.31^{* * *}(0.02)$ & $-0.31^{* * *}(0.03)$ & $-0.36^{* * *}(0.05)$ \\
\hline \multicolumn{4}{|l|}{ Birth Weight (reference not low) } \\
\hline Low birth weight & $-0.19^{* * *}(0.03)$ & $-0.20^{* * *}(0.03)$ & $-0.08(0.06)$ \\
\hline Very low birth weight & $-0.47^{* * *}(0.04)$ & $-0.48^{* * *}(0.05)$ & $-0.50^{* * *}(0.14)$ \\
\hline Age at Bayley testing & $-0.19^{* * *}(0.02)$ & $-0.19^{* * *}(0.03)$ & $-0.20^{* * *}(0.03)$ \\
\hline First born child & $-0.04(0.03)$ & $-0.02(0.04)$ & $-0.05(0.07)$ \\
\hline \multicolumn{4}{|l|}{ Family Covariates } \\
\hline Single mother & $-0.02(0.03)$ & $-0.04(0.04)$ & $-0.21^{* *}(0.08)$ \\
\hline Maternal care at 9 months & $-0.04(0.02)$ & $-0.08^{* *}(0.03)$ & $-0.05(0.05)$ \\
\hline English as Home Language & $0.23^{* * *}(0.03)$ & $0.27^{* * *}(0.04)$ & $0.30^{* *}(0.10)$ \\
\hline Number of siblings & $-0.07^{* * *}(0.01)$ & $-0.04^{*}(0.20)$ & $-0.06(0.03)$ \\
\hline \multicolumn{4}{|l|}{ Maternal Ed (reference HS Diploma) } \\
\hline Less than HS & $-0.06(0.03)$ & $-0.07(0.04)$ & $-0.26^{* *}(0.09)$ \\
\hline Some College & $0.07^{* *}(0.03)$ & $0.06(0.03)$ & $0.02(0.06)$ \\
\hline Bachelor's or Higher & $0.31^{* * *}(0.03)$ & $0.29^{* * *}(0.04)$ & $0.18^{*}(0.07)$ \\
\hline Intercept & $1.24^{* * * *}(0.09)$ & $1.26^{* * *}(0.15)$ & $0.14(0.12)$ \\
\hline$N$ & 8350 & 8350 & 8350 \\
\hline$R^{2}$ & .246 & .218 & .294 \\
\hline
\end{tabular}

Note. Standard errors in parentheses.

* $p<0.05$ 


$$
\begin{array}{rl}
* * & p<0.01, \\
& * * * \\
& p<0.001 .
\end{array}
$$

All covariates were measured at nine months. The dependent variable, prior Bayley score, child's age, and number of siblings are z-scored. An indicator variable for whether a child was missing the Bayley at 9 months was included. ECLS-B weight $W 2 C O$ was used in regression 2. Tests for interactions between care type and poverty status were non-significant, as were tests for interactions between care type and quality (low, medium, and high). Ns were rounded to the nearest 50. 